Int. J. Odontostomat., 11(1):41-46, 2017.

\title{
Evaluación Clínica de Barniz de Flúor en el Manejo de la Hipersensibilidad Dentinaria
}

\author{
Clinical Evaluation Fluoride Varnish in the Management of Dentin Hypersensitivity
}

\author{
Diego Salazar P. ${ }^{1} \&$ Jorge Nakouzi M. ${ }^{2}$
}

SALAZAR, P. D. \& NAKOUZI, M. J. Evaluación clínica de barniz de flúor en el manejo de la hipersensibilidad dentinaria. Int. J. Odontostomat., 11(1):41-46, 2017.

RESUMEN: La Hipersensibilidad Dentinaria (HD) se define como un corto y fuerte dolor generado por la exposición de dentina, que surge de un estímulo químico, térmico, táctil u osmótico, el cual no puede catalogarse como una patología dental propiamente tal. La teoría hidrodinámica de Brännström es la más aceptada en la actualidad para explicar la etiopatogenia de esta afección. Muchos productos han sido elaborados con el fin de combatir la HD, pero no todos constan de la efectividad deseada para atenuarla. El objetivo del presente estudio es evaluar la efectividad inmediata y mediata (posterior a 2 semanas), de un barniz de flúor (Flúor Protector ${ }^{\circledR}$ ) utilizado en la problemática. El estudio es un ensayo clínico no controlado. La muestra consistió de 30 pacientes, sin distinción de género, de entre 20 a 60 años, atendidos en las clínicas de la Facultad de Odontología de la Universidad Andrés Bello, cuyo diagnóstico fue HD, en al menos un diente, con recesión gingival de al menos $2 \mathrm{~mm}$, la cual presento dentina expuesta desde la unión amelocementaria, y además que respondieron a 3 o más puntos en la Escala Numérica (EN), cuando fue aplicado el estímulo evaporativo. Los datos fueron recogidos, en una ficha diseñada para el estudio, la cual incluyo datos del paciente y $9 \mathrm{EN}$, con las cuales se cuantifico la percepción de dolor de los pacientes frente a 3 estímulos: evaporativo, táctil y térmico. Estadísticamente se utilizó análisis de tipo descriptivo y de varianza de Friedman. Se obtuvieron resultados estadísticamente significativos $(p<0.05)$ en la disminución casi completa de la HD frente al tratamiento inmediato (mediana< 3 ), además de la mantenida reducción de la misma en el control posterior a 2 semanas de aplicada la terapia. En conclusión, la terapia con flúor barniz es efectiva en el tratamiento inmediato y mediato de la HD.

PALABRAS CLAVE: hipersensibilidad dentinaria, Escala Numérica (EN), barniz de flúor.

\section{INTRODUCCIÓN}

La hipersensibilidad dentinaria se define como un dolor breve y agudo ocasionado por exposición de la dentina a estímulos de tipo térmicos, táctiles, osmóticos o químicos, que no puede ser atribuido a ninguna forma de patología o defecto dental propiamente tal (Bartold, 2006; Sethna et al., 2011; Cordero García \& Peña Sisto, 2012).

Con respecto a la etiología de la hipersensibilidad dentinaria, se considera de carácter multifactorial (Bartold, 2006). De las muchas teorías presentadas para explicar la etiopatogenia de la problemática en la actualidad, la más aceptada es la
Teoría Hidrodinámica de Brännström (Bartold; Ardila Medina, 2009; Alvarez et al., 2010; Aparna et al., 2010). Existe una gran gama de productos de uso profesional y de uso doméstico para el tratamiento de esta afección.

Si bien muchos productos han sido elaborados con el fin de combatir la hipersensibilidad dentinaria, no todos constan de la efectividad deseada para atenuarla (Sethna et al.), por lo cual este trabajo de investigación pretende evaluar el efecto clínico de un barniz de flúor (Flúor Protector $®$ ) en el manejo de la hipersensibilidad dentinaria.

\footnotetext{
${ }^{1}$ Cirujano dentista Universidad. Andrés Bello, Facultad de odontología, Sede Santiago.

${ }^{2}$ Magíster en odontología, especialista en Rehabilitación Oral U. Andrés Bello, Facultad de odontología U. Andrés Bello, Sede Santiago, Santiago.
} 


\section{MATERIAL Y MÉTODO}

Este estudio fue de tipo analítico, modalidad ensayo clínico no controlado. La muestra consistió de 30 pacientes, sin distinción de género, de entre 20 a 60 años, atendidos en las clínicas de la Facultad de Odontología de la Universidad Andrés Bello, específicamente en los ramos de Clínica Integral del Adulto de IV y $\vee$ año e Internado clínico, cuyo diagnóstico fue hipersensibilidad dental, en al menos un diente, con recesión gingival de al menos $2 \mathrm{~mm}$, la cual presenta dentina expuesta desde la unión amelocementaria, y además que respondieron a 30 más puntos en la escala numérica, cuando fue aplicado el estímulo evaporativo (Sethna et al.) . El diagnóstico de hipersensibilidad dentinaria fue de carácter clínico, y radiográfico, con la finalidad de esclarecer diagnósticos diferenciales. La recesión fue medida con una sonda periodontal Carolina del Norte, marca Hu Friedy ${ }^{\circledR}$. Los criterios de exclusión aplicados a la muestra fueron los siguientes: Pacientes con restauraciones defectuosas, señálese grietas, fracturas o caries marginales; piezas con lesiones de caries, piezas con enfermedad periodontal activa; piezas pilares de prótesis; piezas sometidas a fuerzas ortodóncicas antes de 1 año de alta en el tratamiento; piezas que han recibido terapia periodontal dentro de los últimos 6 meses y piezas con trauma oclusal (Aparna et al.; Sethna et al.). La muestra fue calculada con un error aceptado para el intervalo de confianza del $5 \%$, y con un porcentaje de casos de la muestra de un $98 \%$, siguiendo la fórmula de tamaño de muestra para la estimación de porcentaje.

Los aspectos éticos y legales de esta investigación fueron resguardados, de acuerdo a las exigencias del comité de bioética de la institución. La participación de los pacientes en el estudio fue aceptada luego de la lectura y aprobación y firma del consentimiento informado. El producto utilizado para el estudio fue Flúor Protector ${ }^{\circledR}$, que es un barniz de flúor silano al $1 \%$ en una base de poliuretano, que por cada $\mathrm{ml}$ contiene $1 \mathrm{mg}$ de lon flúor en su estructura, cuya presentación fue la de ampollas de vidrio de 1 $\mathrm{ml}$. El desafió que presentó este estudio fue objetivar una percepción totalmente subjetiva, como lo es el dolor, por lo que fue necesario el uso de herramientas que cumplieran tal propósito. Se realizó una ficha clínica, la cual contenía en su estructura 9 Escalas Numéricas (EN), 3 para la línea basal, 3 para evaluar el tratamiento inmediato, y 3 para evaluar el efecto mediato del tratamiento ( 2 semanas después).
Las 3 escalas estuvieron destinadas a medir de manera individual el dolor percibido en las 3 pruebas de sensibilidad que se aplicaron, en la cual 0 correspondió a ausencia de percepción dolorosa y 10 fue la máxima percepción dolorosa alcanzada. Se midió la percepción dolorosa de los pacientes frente a tres estímulos: estímulo táctil, estímulo térmico y estímulo evaporativo. El estímulo táctil se realizó con la exploración de toda la superficie de la dentina hipersensible (Aparna et al.; Sethna et al.).Para esto se utilizó una sonda de caries a una presión de 70 gramos aproximadamente (Sethna et al.). El estímulo térmico se llevó a cabo en la pieza a estudiar sin presencia de fluidos orales en la zona sensible. Para esto se utilizó aislamiento relativo con tórulas de algodón. Para el estímulo se utilizó agua fresca desmineralizada, obtenida de los dispensadores de la facultad, y refrigerada a $10^{\circ} \mathrm{C}$ (Sethna et al.) en una jeringa de $60 \mathrm{ml}$ marca $\mathrm{MT}^{\circledR}$. Para tales efectos se ocuparon los refrigeradores dispuestos en los botiquines de la clínica odontológica de la facultad, y la temperatura fue monitorizada por un termómetro digital. Esta agua fue transportada en un recipiente aislante de plumavit, al momento de ser sacada la jeringa con agua del refrigerador. El agua volvió a ser refrigerada cuando terminaron las pruebas entre pacientes. El agua se aplicó con una motita de algodón, la cual fue embebida en agua, y se aplicó en toda la superficie expuesta de la dentina hipersensible (Aparna et al.; Sethna et al.). El estímulo evaporativo se llevó a cabo con la aplicación de un chorro de aire suministrado con la jeringa triple, orientada de manera perpendicular a la superficie expuesta de la dentina, a una distancia de $1 \mathrm{~cm}$, por el lapso de 1 segundo (Aranha et al., 2009; Hoang-Dao et al., 2009; Aparna et al.; Sethna et al.). La aplicación de los tres estímulos buscó generar cambios osmóticos en los túbulos dentinarios, generando movimiento en el fluido al interior de ellos, gatillando el estímulo. Los estímulos fueron aplicados en el orden establecido en la ficha clínica diseñada para el estudio. Cada estímulo fue aplicado en el mismo diente, esperando $5 \mathrm{mi}-$ nutos (Hoang-Dao et al.; Aparna et al.) entre cada test, tiempo necesario para que la pulpa dentaria llegue a un equilibrio fisiológico. El tiempo fue medido por un cronómetro. En relación a los estímulos ocupados en el estudio, el estímulo evaporativo, táctil y térmico son recomendables ya que son fisiológicos y controlables. Por otro lado existen muchos estímulos que pueden causar hipersensibilidad dentinaria, pero 
algunos de estos son poco prácticos de emplear clínicamente (Hoang-Dao et al.). Con respecto al tiempo transcurrido entre las mediciones (5 minutos), este era mucho más largo que el periodo refractario de los nervios sensoriales (Hoang-Dao et al.). Los datos obtenidos correspondieron a la medición, los cuales fueron constatados en la ficha diseñada para el estudio. Posterior a esto se aplicó Flúor Protector ${ }^{\circledR}$, con un tip, en la misma pieza, específicamente en la zona de exposición cervical, para luego volver a realizar las mediciones de los 3 estímulos, constatando en la EN de la ficha la percepción que obtuvieron los pacientes, después de aplicado el producto en estudio. Con esto se buscó evaluar el efecto inmediato que poseía el producto en el tratamiento de la hipersensibilidad. Después de esto, el paciente fue entrenado para no usar ningún producto desensibilizante de uso doméstico hasta la siguiente sesión de estudio, o sea 2 semanas después (Ritter et al., 2006; Hoang-Dao et al.;), con el fin de no alterar los resultados. Concluidas las 2 semanas de espera, se procedió a aplicar los test nuevamente, y así se observó el efecto mediato del material en estudio. Todos los datos obtenidos fueron consignados en la ficha clínica diseñada para el estudio. Una vez obtenidos todos los datos de la muestra, se procedió a numerar cada ficha, en orden aleatorio, del 1 al 30, para así tener un orden de referencia en la tabulación de los resultados en planilla Excel. El método mediante el cual se midió la efectividad de este producto fue comparando la disminución en los valores de la EN; antes, después del tratamiento y en el control posterior a 2 semanas, y también con la disminución de los valores de la EN bajo 3 puntos, ya que este era uno de los criterios de inclusión para catalogar la percepción del paciente como hipersensibilidad dentinaria frente al estímulo de selección (evaporativo) (Sethna et al.). Con respecto al tipo de análisis que se utilizó, los datos primarios fueron sometidos a la prueba de normalidad de Shapiro-Wilk (Shapiro \& Wilk, 1965) y a la prueba de Levene, para determinar homoscedasticidad (igualdad de varianza). Posteriormente, estos datos fueron sometidos a estudios descriptivos y se estimaron los estadígrafos mediana y percentil 25 y 75 (Ostle, 1980; Díaz, 2009). La distribución de los datos se dibujó en gráficos de barras con el valor de la mediana (Díaz). Para finalizar el análisis estadístico, los momentos en cada una de las variables analizadas fueron comparados mediante un análisis de varianza de Friedman, previa estimación de los rangos que presentaron los datos de cada tratamiento. El Nivel de Significación fue de a = 0,05 .

\section{RESULTADOS}

Los resultados de la aplicación de las pruebas de Shapiro-Wilk y Levene, en todos los casos, fueron altamente significativas $(p<0,01)$, lo que indica que los datos no se distribuyen normalmente y los momentos comparados no se caracterizan por cumplir con la propiedad de homoscedasticidad (igualdad de varianzas). En relación a la hipersensibilidad dentinaria provocada por el estímulo evaporativo, el tratamiento inmediato muestra resultados estadísticamente significativos en la supresión de la hipersensibilidad dentinaria (mediana < 3 ), al igual que en el control después de 2 semanas, donde se aprecian resultados positivos en la reducción de la hipersensibilidad, pero con un leve retorno de la problemática.

Según el análisis Friedman, todos los valores de la EN se diferencian entre los tres momentos estudiados $(p<0,05)$, lo cual indica que existen diferencias estadísticamente significativas entre los valores de la hipersensibilidad antes del tratamiento, en comparación al post-tratamiento y a 2 semanas después de ser aplicada la terapia.

En relación a la hipersensibilidad dentinaria provocada por el estímulo táctil, el tratamiento inmediato muestra resultados estadísticamente significativos en la supresión de la hipersensibilidad dentinaria (mediana $=0$ ), al igual que en el control posterior a 2 semanas, donde se aprecia permanencia de la mejoría obtenida en el tratamiento inmediato (mediana $<3$ ).

Según el análisis Friedman, todos los valores de la EN se diferencian entre los tres momentos estudiados $(p<0,05)$, lo cual indica que existen diferencias estadísticamente significativas entre los valores de la hipersensibilidad antes del tratamiento, en comparación al post-tratamiento y a 2 semanas después de ser aplicada la terapia.

En relación a la hipersensibilidad dentinaria provocada por el estímulo térmico, el tratamiento inmediato muestra resultados estadísticamente significativos en la supresión de la hipersensibilidad dentinaria (mediana $<3$ ), al igual que en el control después de 2 semanas, donde se aprecian resultados positivos en la reducción de la hipersensibilidad, pero con un leve retorno de la problemática. 
Según el análisis Friedman, todos los valores de la EN se diferencian entre los tres momentos estudiados $(p<0.05)$, lo cual indica que existen diferencias estadísticamente significativas entre los valores de la hipersensibilidad antes del tratamiento, en comparación al post-tratamiento y a 2 semanas después de ser aplicada la terapia.

\section{DISCUSIÓN}

La hipersensibilidad dentinaria es un problema clínico importante en odontología, porque afecta a un gran porcentaje de la población, y como tal, basándonos en la literatura existente, no hay un tratamiento universalmente aceptado, o que sea totalmente efectivo, para su completa abolición (Ritter et al.; Hoang-Dao et al.; Sethna et al.). En las bases de la teoría hidrodinámica propuesta por Brännström, el movimiento rápido de linfa dentinaria al interior de los túbulos dentinarios es capaz de activar terminaciones nerviosas interdentales, y así causar la sensibilidad (Aranha et al., 2009; Ardila Medina, 2009; Bartold, 2006; Alvarez et al., 2010; Sethna et al., 2011) por lo que muchas de las formas de tratamiento están orientadas a reducir la permeabilidad dentinaria, mediante el uso de agentes oclusores de los túbulos dentinarios (Hoang-Dao et al.). Por otra parte, las terapias mediante agentes desensibilizantes de la pulpa, son muy utilizadas en el ámbito odontológico, con buenos resultados clínicos. Las terapias mucogingivales de recubrimiento cervical son otra alternativa de tratamiento para esta afección, al igual que las terapias con láser y la iontoforesis (Ardila Medina). La impregnación de los túbulos dentinarios con resinas plásticas y material adhesivo para sellar los túbulos se ha sugerido en la luz de la teoría hidrodinámica (Panduric et al., 2001). Por otra parte, los clínicos han intentado varias estrategias para tratar esta problemática, incluyendo la aplicación de fluoruros tópicos, que se han utilizado durante al menos 60 años, contra la hipersensibilidad dentinaria (Ritter et al.; Hoang-Dao et al.). El mecanismo de acción de los fluoruros tópicos es crear una barrera mediante la precipitación de $\mathrm{CaF}_{2}$ en la superficie expuesta de la dentina, generando la oclusión de los túbulos expuestos, reduciendo la permeabilidad en la dentina y, en consecuencia, la supresión de la hipersensibilidad (Ritter et al.; Aranha et al.; HoangDao et al.). Por otra parte, las resinas naturales contenidas en los barnices de flúor podrían proporcionar una especie de barrera mecánica adicional, la cual ocluya los túbulos expuestos y ayude a contener las sales de $\mathrm{CaF}_{2}$, impidiendo su disolución en el medio, aunque esto no ha sido probado experimentalmente (Ritter et al.; Hoang-Dao et al.).

Los resultados de este estudio fueron altamente significativos estadísticamente, e indican la efectividad que posee el producto en estudio al eliminar, posterior a su aplicación, de manera inmediata, la hipersensibilidad dentinaria producida por los tres estímulos estudiados: estimulo evaporativo, táctil y térmico, al igual que Hoang-Dao et al., en el cual se ocupó un barniz de flúor al $5 \%$ (Shellac $\mathrm{F}^{\circledR}$ ), y Camilotti et al. (2012) que encontró resultados significativos en la disminución de la hipersensibilidad dentinaria, mediante el uso de barniz de flúor al $2 \%$, después de la primera aplicación. Cabe destacar que en estos estudios se ocupó un barniz de flúor con mayor concentración que el Flúor Protector ${ }^{\circledR}(1 \%)$. Independiente de la concentración de flúor en la fórmula del barniz, el éxito de la terapia es más atribuido al mecanismo de acción del mismo, el cual generaría una barrera que ocluye de manera satisfactoria los túbulos dentinarios, disminuyendo la permeabilidad de la dentina, y además impidiendo la disolución de este en el medio oral, debido a la base del poliuretano, que podría actuar como barrera protectora de las sales de CaF2 (Ritter et al.; Hoang-Dao et al.). Posterior a 2 semanas de aplicación del barniz, se observaron resultados estadísticamente significativos, que revelaron permanencia en la disminución de la sintomatología percibida por los pacientes, lograda posterior a la aplicación del barniz, para los estímulos evaporativo y térmico. Se encontró, de todos modos una recidiva de la hipersensibilidad dentinaria, frente a estos 2 estímulos, la cual sigue siendo inferior en relación al éxito inmediato de la terapia. Con respecto a la sensibilidad percibida en el estímulo táctil, esta se mantuvo baja, en relación al momento de la aplicación de la terapia, posterior a 2 semanas en el control. Que este haya sido el estímulo que mantuvo bajos niveles de sensibilidad puede deberse a que es considerado como el estímulo menos invasivo de todos los empleados (Hoang-Dao et al.), otorgando menor respuesta que otros estímulos. Castillo y Milgrom mostraron que después de la aplicación tópica de $\mathrm{NaF}$ al $5 \%$, la mayor parte del ion fluoruro fue liberado en plazos de 2 semanas, para luego ser liberadas pequeñas cantidades hasta 21 semanas post-tratamiento (Ritter et al.; Hoang-Dao et al.). Esto podría relacionarse a los resultados obtenidos en nuestro estudio, debido a que el control realizado se extendió hasta las 2 
semanas post-tratamiento, tiempo en el cual aún se observan resultados significativos en la reducción de la hipersensibilidad para los 3 estímulos en estudio.

El posterior retorno de la sensibilidad en los estímulos evaporativo y térmico, podría estar influenciado por la remoción mecánica del barniz, que generaría la permeabilización parcial de la dentina. Esto concuerda con lo mencionado por Corona et al. (2003), el cual señala que el uso de barniz de flúor solo conduce a una obliteración parcial de los túbulos dentinarios, observándose además la eliminación del agente desensibilizante durante el cepillado dental. Hansen reporto el éxito de la terapia en base a barniz de $\mathrm{NaF}$ al $5 \%$, con resultados de éxito de un 48 $\%$, en un periodo de 3 meses, en la reducción de la hipersensibilidad dentinaria (Ritter et al.). Si bien los resultados de Hansen son exitosos, cabe hacer presente que Hansen ocupa una escala subjetiva para sus mediciones, además de un flúor barniz con mayor concentración de flúor en su fórmula. HoangDao et al., encontró resultados positivos en la reducción de la hipersensibilidad dentinaria, mediante el uso de varios barnices de flúor (Fluorniz ${ }^{\circledR}$, Duraphat ${ }^{\circledR}$, Duoluorid $X I^{\circledR}$ y Fluorphat ${ }^{\circledR}$ ), los cuales al compararlos, no presentaron diferencias estadísticamente significativas en sus resultados (Camilotti et al.). Esto ratifica que el éxito clínico de la terapia en base a barnices de flúor, se debería al mecanismo de acción que estos poseen, no siendo tan relevante la concentración de ion flúor que estos posean en su fórmula. Existen resultados de otros estudios que indican que con una sola aplicación de barniz de flúor tópico se produciría la reducción de la hipersensibilidad dentinaria durante 24 semanas (Ritter et al.), lo cual no se podría determinar en el presente estudio, debido a que el tiempo de seguimiento fue inferior (2 semanas).

SALAZAR, P. D. \& NAKOUZI, M. J. Clinical evaluation fluoride varnish in the management of dentin hypersensitivity. Int. J. Odontostomat., 11(1):41-46, 2017.

ABSTRACT: Dentin Hypersensitivity (DH) is defined as a short, sharp pain generated by exposure of dentin, which arises from a chemical stimuli, thermal, tactile or osmotic, which can't be categorized as a dental pathology as such. Brännström hydrodynamic theory is widely accepted today to explain the pathogenesis of this condition. Many products have been developed to combat $\mathrm{DH}$, but not all consist of the desired effectiveness to attenuate. The aim of the present study is to evaluate the effectiveness immediate and mediate (after two weeks), a fluoride varnish
(Fluor Protector ${ }^{\mathrm{TM}}$ ), used in the management of patients with this problem. The present study is an uncontrolled clinical trial. The sample consisted of 30 patients, regardless of gender, aged 20 to 60 , treated at the clinics of the Faculty of Dentistry at the "Universidad Andres Bello", whose diagnosis was $\mathrm{DH}$, in a least one tooth with gingival recession at least $2 \mathrm{~mm}$, which exposed dentin present from the CEJ, and also responding to three of more points on the Numeric Scale (NS), when the stimulus was applied evaporative. Data were collected after approval and written informed consent, in a form designed for the study, which included data from the patient and 9 NS, which was quantified with pain perception of patients versus 3 stimuli: evaporative, touch and heat. For statistical analysis we used descriptive analysis and variance of Friedman. Regarding the 3 stimuli under study results were statistically significant $(p<0.05)$ decrease in the almost complete $\mathrm{DH}$ versus immediate treatment (median $<3$ ), besides the maintained reduction thereof in the inspection after 2 weeks of therapy applied. In conclusion, treatment with fluoride varnish is effective in treating the immediate and mediate $\mathrm{DH}$.

KEY WORDS: dentin hypersensitivity, Numeric Scale (NS), fluoride varnish.

\section{REFERENCIAS BIBLIOGRÁFICAS}

Alvarez, C.; Arroyo, P.; Aranguiz, V.; Chaparro, A.; Contreras, R.; Leighton, C.; Moncada, G.; Quintana, M.; Rudolph, M.; Silva, A.; Sommariva, C.; Villavicencio, J. J. \& Xaus, G. Diagnóstico y tratamiento de la hipersensibilidad dentinaria. Rev. Dent. Chile, 101(2):17-25, 2010.

Aparna, S.; Setty, S. \& Thakur, T. Comparative efficacy of two treatment modalities for dentinal hypersensitivity: a clinical trial. Indian J. Dent. Res., 21(4):544-8, 2010.

Aranha, A. C.; Pimenta, L. A. \& Marchi, G. M. Clinical evaluation of desensitizing treatments for cervical dentin hypersensitivity. Braz. Oral Res., 23(3):333-9, 2009.

Ardila Medina, C. M. Hipersensibilidad dentinal: Una revisión de su etiología, patogénesis y tratamiento. Av. Odontoestomatol., 25(3):137-46, 2009.

Bartold, P. M. Dentinal hipersensitivity: a review. Aust. Dent. J., 51(3):212-8, 2006.

Camilotti, V.; Zilly, J.; Busato Pdo., M.; Nassar, C. A. \& Nassar, P. O. Desensitizing treatments for dentin hypersensitivity: a randomized, split-mouth clinical trial. Braz. Oral Res., 26(3):2638, 2012.

Cordero García, S. \& Peña Sisto, M. Factores de riesgo de hipersensibilidad dentinaria en pacientes adultos con prótesis dental. MEDISAN, 16(3):349-57, 2012.

Corona, S. A.; Nascimento, T. N.; Catirse, A. B.; Lizarelli, R. F.; Dinelli, W. \& Palma-Dibb, R. G. Clinical evaluation of low-level laser therapy and fluoride varnish for treating cervical dentinal hypersensitivity. J. Oral Rehabil., 30(12):1183-9, 2003.

Díaz, V. Metodología de la Investigación Científica y Bioestadística para Profesionales y Estudiantes de Ciencias Médicas. Santiago de Chile, RiL Editores, 2009.

Hoang-Dao, B. T.; Hoang-Tu, H.; Tran-Thi, N. N.; Koubi, G.; Camps, 
J. \& About, I. Clinical efficiency of a natural resin fluoride varnish (Shellac F) in reducing dentin hypersensitivity. J. Oral Rehabil., 36(2):124-31, 2009.

Ostle, B. Estadística Aplicada. La Habana, Editorial Científico-Técnica, 1980.

Panduric', V.; Knezevic', A.; Tarle, Z. \& Sutalo, J. The efficiency of dentine adhesives in treating non-caries cervical lesions. J. Oral Rehabil., 28(12):1168-74, 2001.

Ritter, A. V.; de L. Dias, W.; Miguez, P.; Caplan, D. J. \& Swift, E. J. Jr. Treating cervical dentin hypersensitivity with fluoride varnish: a randomized clinical study. J. Am. Dent. Assoc., 137(7):1013-20, 2006.

Sethna, G. D.; Prabhuji, M. L. \& Karthikeyan, B. V. Comparison of two different forms of varnishes in the treatment of dentine hypersensitivity: a subject-blind randomised clinical study. Oral Health Prev. Dent., 9(2):143-50, 2011

Shapiro, S. S. \& Wilk, M. B. An analysis of variance test for normality (complete samples). Biometrika, 52(3-4):591-611, 1965.
Dirección para correspondencia:

Diego Salazar P.

Cirujano dentista Universidad Andrés Bello

Facultad de odontología

Sede Santiago.

Santiago

E-mail:disap09.dsp@gmail.com

Recibido: 13-07-2016

Aceptado: 23-12-2016 